\title{
A New Algorithm for the Verification of Codiagnosability of Discrete Event Systems With Dynamic Observations *
}

\author{
Wesley R. Silveira* Marcos V. Moreira* \\ * Cidade Universitária, Av. Athos da Silveira Ramos, 149 - CT, \\ Universidade Federal do Rio de Janeiro, Rio de Janeiro, RJ, Brazil \\ (wesley08@poli.ufrj.br/moreira.mv@poli.ufrj.br).
}

\begin{abstract}
:
In this work, a new algorithm for verifying the codiagnosticability of the language of a discrete event system (DES) with dynamic observations is proposed. The new algorithm is an extension of a recently proposed algorithm for the verification of the codiagnosability of DES with static observations. The computational complexity of the proposed algorithm is compared with the complexity of other algorithms presented in the literature.
\end{abstract}

Keywords: Diagnosability, Discrete Event Systems, Dynamic Observation, Verifiers.

\section{INTRODUCTION}

Several works presented in the literature address the problem of fault diagnosis of Discrete Event Systems (DES) (Sampath et al., 1995; Qiu and Kumar, 2006; Moreira et al., 2011; Cabral et al., 2015). The fault diagnosis consists of identifying the occurrence of an unobservable fault event within a bounded number of event occurrences, based on the observation of the system events. The fault diagnosis can be carried out by using a centralized or a decentralized architecture. In the centralized case a monolithic diagnoser is computed to identify the fault occurrence, whereas, in the decentralized case, a set of local diagnosers and a coordinator are used to detect the fault.

Different protocols have been proposed for the diagnosis of DES in the decentralized case (Debouk et al., 2000). In the simplest protocol, each local diagnoser has its own set of observable events, and the diagnosers do not communicate among each other. When a fault is detected by a local diagnoser, it sends this information to the coordinator that issues an alert to the operator of the system. The property of the system language that guarantees that a fault event can always be diagnosed within a bounded number of event occurrences is called codiagnosability (Qiu and Kumar, 2006).

In the majority of the works proposed in the literature (Sampath et al., 1995; Moreira et al., 2011; Cabral et al., 2015; Debouk et al., 2000; Contant et al., 2006; Qiu and Kumar, 2006; Jiang et al., 2001; Kilic, 2008; Zad et al., 2005), the event observations are static in the sense that they do not depend on the trace executed by the system or its current state. However, in some cases, the observation of the system events are dynamic, i.e.,

\footnotetext{
‡ This work has been partially supported by the Brazilian Research Council (CNPq) under grants 305267/2018-3 and 431307/20180 , and the Coordenação de Aperfeiçoamento de Pessoal de Nível Superior - Brasil (CAPES) Finance Code 001.
}

their observations depend on the transition or the trace executed by the system (Thorsley and Teneketzis, 2007; Cassez and Tripakis, 2008; Wang et al., 2010a,b). For instance, the observation of the system events is dynamic in sensor activation problems where a local agent can turn on/off sensors during the evolution of the system motivated by different reasons, including sensors with limited energy, limited bandwidth for communication, or security issues. In these cases, the verification of the codiagnosability of the system cannot be carried out by using the same verifiers proposed for DES with static observations, such as the verification methods proposed in Qiu and Kumar (2006); Yoo and Lafortune (2002); Moreira et al. (2011).

The verification of codiagnosability of DES with dynamic observations is addressed in Wang et al. (2011); Yin and Lafortune (2015). In Wang et al. (2011), a verifier called C-VERIFIER, which is a nondeterministic automaton constructed using the notion of cluster automata, is proposed. Since, as shown in Wang et al. (2011), the number of states of the C-VERIFIER is $O\left(|X|^{n+1}\right)$, where $n$ is the number of local agents, and it is a nondeterministic automaton, then the complexity for the computation of the C-VERIFIER is $O\left(|X|^{2(n+1)} \times|\Sigma|\right)$. The verifier presented in Yin and Lafortune (2015), called T-VERIFIER, is an extension of the standard verifier approach used for static diagnosis presented in Jiang et al. (2001); Yoo and Lafortune (2002) to the case of dynamic observations. The T-VERIFIER is a deterministic automaton generated by using a set of rules in which event tuples are used to label the transitions, and the transition function is defined by computing four sets of local agents for each one of its transitions, according to the dynamic observation of the system events. As shown in Yin and Lafortune (2015), the number of states of the T-VERIFIER is in the worst-case equal to $|X|^{n+1}$, and the computational complexity of the T-VERIFIER is $O\left(n \times|X|^{n+1} \times|\Sigma|\right)$. 
In this technical note, we present an efficient algorithm for the verification of the codiagnosability of DES with dynamic observations. The proposed verifier, called in this paper as E-VERIFIER, is an extension of the verification method presented in Moreira et al. (2011) to the case of dynamic observations. As in Moreira et al. (2011), an event renaming function is used, and differently from Yin and Lafortune (2015), it is not needed to compute sets of local agents for each possible transition of the verifier to define its transition function. The conditions associated with the dynamic observations of the system events are added directly to the automata that model the fault-free behavior and the faulty behavior of the system, reducing the computational cost for codiagnosability verification.

The E-VERIFIER becomes equal to the verifier for the static case presented in Moreira et al. (2011) if dynamic observations are replaced with static observations. It is also shown in this paper that, although the proposed algorithm leads to a smaller computational cost than the algorithm proposed in Yin and Lafortune (2015) when the size of the verifier grows, the computational complexities of the E-VERIFIER and the T-VERIFIER are the same.

This paper is organized as follows. In Section 2 we present some preliminary concepts and introduce the notion of codiagnosability with dynamic observations. In Section 3 we provide an algorithm for the computation of the E-VERIFIER. In Section 4, we show the computational complexity of the verification algorithm. The conclusions are drawn in Section 5.

\section{PRELIMINARIES}

\subsection{Notations and definitions}

Let $G=\left(X, \Sigma, f, x_{0}\right)$ be the automaton model of a DES, where $X$ is the set of states, $\Sigma$ is the finite set of events, $f: X \times \Sigma \rightarrow X$ is the partial transition function, and $x_{0}$ is the initial state.

The transition function $f$ is extended to $X \times \Sigma^{*}$ in the usual way, where $\Sigma^{*}$ denotes the Kleene-closure of $\Sigma$ (Cassandras and Lafortune, 2008). Since $f$ is a partial function, then the language generated by $G$ can be defined as $\mathcal{L}(G)=L=\left\{s \in \Sigma^{*}: f\left(x_{0}, s\right)\right.$ is defined $\}$, and the transition set of $G$ is defined as $T R(G)=\{(x, \sigma) \in X \times$ $\Sigma: f(x, \sigma)$ is defined $\}$. The prefix-closure of a language $L$ is given by $\bar{L}=\left\{s \in \Sigma^{*}:\left(\exists t \in \Sigma^{*}\right) \wedge(s t \in L)\right\}$.

The accessible and the coaccessible part of $G$ are denoted by $A c(G)$ and $\operatorname{CoAc}(G)$, respectively.

Let $G_{1}$ and $G_{2}$ be two automata. Then $G_{1} \times G_{2}$ and $G_{1} \| G_{2}$ denote the completely synchronous and the synchronous compositions of $G_{1}$ and $G_{2}$, respectively (Cassandras and Lafortune, 2008).

The active event function of $G$ is defined as $\Gamma_{G}: X \rightarrow 2^{\Sigma}$, where $\Gamma_{G}(x)=\{\sigma \in \Sigma: f(x, \sigma)$ is defined $\}$.

With a slight abuse of notation, $\sigma \in s$ denotes that $\sigma \in \Sigma$ is one of the events that form trace $s \in \Sigma^{*}$.

The static projection operation $P_{s}^{l}: \Sigma_{l}^{*} \rightarrow \Sigma_{s}^{*}$, where $\Sigma_{s} \subset \Sigma_{l}$ is defined as $P_{s}^{l}(\varepsilon)=\varepsilon$, where $\varepsilon$ is the empty trace, and $P_{s}^{l}(\sigma)=\sigma$, if $\sigma \in \Sigma_{s}$ or $P_{s}^{l}(\sigma)=\varepsilon$, if $\sigma \in \Sigma_{l} \backslash \Sigma_{s}$, and $P_{s}^{l}(s \sigma)=P_{s}^{l}(s) P_{s}^{l}(\sigma)$, for all $s \in \Sigma_{l}^{*}$, and $\sigma \in \Sigma_{l}$. The static projection can also be applied to language $L$, by applying the projection to all traces $s \in L$. The inverse projection $P_{s}^{l^{-1}}: \Sigma_{s}^{*} \rightarrow 2^{\Sigma_{l}^{*}}$ when applied to a trace $s \in \Sigma_{s}^{*}$ generates all traces of $\Sigma_{l}^{*}$ whose projection is equal to $s$. The inverse projection can also be applied to languages.

In this paper we consider the decentralized diagnosis scheme composed of $n$ local agents, that do not communicate among each other, and a coordinator. In this scheme, a fault is diagnosed when at least one of the local agents detects and communicates its occurrence to the coordinator. Let $A=\{1, \ldots, n\}$ be the index set of the local agents. In the case of decentralized observations, there exists a subset of observable events $\Sigma_{o_{i}} \subset \Sigma$, associated with each agent $i \in A$, where $\Sigma_{o_{i}} \cap \Sigma_{o_{j}}$ can be different from the empty set, for $i, j \in A, i \neq j$. When dynamic observations are considered, an event $\sigma \in \Sigma_{o_{i}}$ can be observed or not by local agent $i \in A$, depending on the current state or the trace executed by the system. Thus, the set $\Sigma_{o_{i}}$ is composed of all events that can be observed after at least one of their occurrences by local agent $i$. Consequently, $\Sigma_{o}=\bigcup_{i=1}^{n} \Sigma_{o_{i}}$ is the set of events that are potentially observable by at least one local agent, and $\Sigma_{u o}=\Sigma \backslash \Sigma_{o}$ is the set of completely unobservable events of $G$.

Dynamic observations are considered in the literature in two different ways (Cassez and Tripakis, 2008): (i) language-based; and ( $i i)$ transition-based. In the languagebased dynamic observation, the observability of an event $\sigma \in \Sigma$, associated with a given transition $(x, \sigma) \in T R(G)$, depends on the sequence of events $s \in L$ that is executed by the system to reach state $x=f\left(x_{0}, s\right)$. To characterize the language-based dynamic observation let us define the observation mapping $\omega_{i}: L \rightarrow 2^{\Sigma_{o_{i}}}, i \in A$, that associates with each $s \in L$, a subset of $\Sigma_{o_{i}}$ corresponding to the events that are observable after the occurrence of trace $s$. Thus, event $\sigma$ associated with transition $(x, \sigma)$ is observable if $\sigma \in \omega_{i}(s)$. On the other hand, in the transitionbased dynamic observation, the observation of an event $\sigma$ associated with a transition $(x, \sigma)$ is independent of the trace executed to reach state $x$. In the transition-based dynamic observation, all traces $s \in L$ that leads to the same state $x \in X$, have the same observation mapping, i.e., $\forall s, t \in L$, if $f\left(x_{0}, s\right)=f\left(x_{0}, t\right)$, then $\omega_{i}(s)=\omega_{i}(t)$. In this paper, we consider only transition-based dynamic observations.

In order to define a projection operation taking into account dynamic observations of events, called here, for simplicity, dynamic projection, it is necessary to define the set of observable transitions for agent $i \in A$ as $\Omega_{i}=$ $\left\{(x, \sigma) \in T R(G):(\exists s \in L)\left[f\left(x_{0}, s\right)=x\right] \wedge\left[\sigma \in \omega_{i}(s)\right]\right\}$. Then, the dynamic projection $P_{\Omega_{i}}: L \rightarrow \Sigma_{o_{i}}^{*}$ can be computed recursively as follows: $P_{\Omega_{i}}(\varepsilon)=\varepsilon$, and for all $s \in L$ and $s \sigma \in L$, where $\sigma \in \Sigma$,

$$
P_{\Omega_{i}}(s \sigma)=\left\{\begin{array}{l}
P_{\Omega_{i}}(s) \sigma, \text { if }\left(f\left(x_{0}, s\right), \sigma\right) \in \Omega_{i} \\
P_{\Omega_{i}}(s), \text { if }\left(f\left(x_{0}, s\right), \sigma\right) \notin \Omega_{i} .
\end{array}\right.
$$

Note that the dynamic projection $P_{\Omega_{i}}$ becomes equal to a static projection $P_{o_{i}}: \Sigma^{*} \rightarrow \Sigma_{o_{i}}^{*}$ if all events $\sigma \in \Sigma$ that are observable after a given sequence $s \in L$, are also observable after any sequence $s^{\prime} \in L$ such that $\sigma \in \Gamma_{G}(x)$ and $x=f\left(x_{0}, s^{\prime}\right)$. Thus, the static projection $P_{o_{i}}$ is a special case of the dynamic projection $P_{\Omega_{i}}$. 


\subsection{Diagnosis under dynamic observations}

Fault diagnosis is the process of identifying the occurrence of an unobservable fault event, within a bounded number of observations of events generated by the system. We assume, without loss of generality, that the language generated by automaton $G, L$, is live, i.e., $\Gamma_{G}(x) \neq \emptyset$ for all $x \in X$. For the sake of simplicity, it is also assumed that there is only one fault event, i.e., $\Sigma_{f}=\left\{\sigma_{f}\right\}$. Thus, a fault trace is a sequence of events $s$ such that $\sigma_{f} \in s$. A fault-free trace $\omega$, on the other hand, does not contain the fault event $\sigma_{f}$, i.e., $\sigma_{f} \notin \omega$.

Let $G_{N}$ be the subautomaton of $G$ that represents the fault-free behavior of the system with respect to the fault event set $\Sigma_{f}$, i.e., the language generated by $G_{N}$ is $L_{N} \subset L$ formed of all fault-free traces generated by the system. Thus, the set of all fault traces generated by the system is $L \backslash L_{N}$, where \denotes set difference.

In the sequel, we present the definition of codiagnosability considering static observations of events presented in Debouk et al. (2000).

Definition 1. Let $L$ be the prefix-closed language generated by automaton $G$, and let $L_{N} \subset L$ be the prefixclosed language formed of all fault-free traces generated by the system. Then, $L$ is said to be codiagnosable w.r.t. projection $P_{o_{i}}: \Sigma^{*} \rightarrow \Sigma_{o_{i}}^{*}, i \in A$, and $\Sigma_{f}$ if:

$$
\begin{gathered}
(\exists z \in \mathbb{N})\left(\forall s \in L \backslash L_{N}\right)\left(\forall s t \in L \backslash L_{N},\|t\| \geq z\right) \Rightarrow \\
(\exists i \in A)\left(P_{o_{i}}(s t) \neq P_{o_{i}}(\omega), \forall \omega \in L_{N}\right),
\end{gathered}
$$

where ||.|| denotes the length of a trace.

In the context of dynamic observations, the definition of codiagnosability is given as follows (Wang et al., 2011; Yin and Lafortune, 2015).

Definition 2. Let $L$ be the prefix-closed language generated by automaton $G$, and let $L_{N} \subset L$ be the prefixclosed language formed of all fault-free traces generated by the system. Then, $L$ is said to be codiagnosable w.r.t. the dynamic projections $P_{\Omega_{i}}: L \rightarrow \Sigma_{o_{i}}^{*}, i \in A$, and $\Sigma_{f}$ if:

$$
\begin{gathered}
(\exists z \in \mathbb{N})\left(\forall s \in L \backslash L_{N}\right)\left(\forall s t \in L \backslash L_{N},\|t\| \geq z\right) \Rightarrow \\
(\exists i \in A)\left(P_{\Omega_{i}}(s t) \neq P_{\Omega_{i}}(\omega), \forall \omega \in L_{N}\right) .
\end{gathered}
$$

Note that the unique difference between Definitions 1 and 2 is the projection operation, which in the former definition is static, whereas in the latter is dynamic.

\section{VERIFICATION OF CODIAGNOSABILITY WITH DYNAMIC OBSERVATIONS}

We present in the sequel a new algorithm for the verification of codiagnosability with dynamic observations, based on the algorithm for codiagnosability verification for systems with static observations presented in Moreira et al. (2011). In order to do so, it is first necessary to compute the automaton that models the fault-free behavior of the system $G_{N}$, and the automaton that models the faulty behavior $G_{F}$, i.e., the automaton whose generated language is $\overline{L \backslash L_{N}}$. Automaton $G_{N}$ is computed by eliminating from $G$ all transitions labeled with $\sigma_{f}$ and taking its accessible part. Automaton $G_{F}$ can be computed by following three steps (Moreira et al., 2011): (i) compute the synchronous composition of $G$ with the label automaton $A_{l}=\left(\{N, F\},\left\{\sigma_{f}\right\}, f_{l},\{N\}\right)$, where $f_{l}\left(N, \sigma_{f}\right)=F$ and $f_{l}\left(F, \sigma_{f}\right)=F$; $(i i)$ mark the states labeled with $F$; and (iii) take the coaccessible part of the resulting automaton. For simplicity, we present the verification method considering only two local agents.

Algorithm 1. Verification of codiagnosability with dynamic observations

Input: $G, \Omega_{i}$, for $i \in A=\{1,2\}$, and $\Sigma_{f}=\left\{\sigma_{f}\right\}$.

Output: Codiagnosability decision

1: Compute automaton $G_{N}=\left(X_{N}, \Sigma_{N}, f_{N}, x_{0}\right)$, where $\Sigma_{N}=\Sigma \backslash \Sigma_{f}$

2: Compute the fault automaton $G_{F}=\left(X_{F}, \Sigma, f_{F}\right.$, $\left.\left(x_{0}, N\right)\right)$ as presented in Moreira et al. (2011).

3: Compute automaton $G_{F I}$ from $G_{F}$ as follows: 3.1: Define function $I: T R\left(G_{F}\right) \rightarrow 2^{A}$, as:

$$
I\left(x_{F}, \sigma\right)=\left\{i \in A:(x, \sigma) \in \Omega_{i}\right\},
$$

where $x_{F}=(x, l)$, with $l \in\{F, N\}$.

3.2: Construct automaton $G_{F I}=\left(X_{F}, \Sigma_{F I}, f_{F I}\right.$, $\left.\left(x_{0}, N\right)\right)$, where $\Sigma_{F I}=\left\{\sigma^{I\left(x_{F}, \sigma\right)}:\left(x_{F}, \sigma\right) \in\right.$ $\left.T R\left(G_{F}\right)\right\}$ and $f_{F I}\left(x_{F}, \sigma^{I\left(x_{F}, \sigma\right)}\right)=f_{F}\left(x_{F}, \sigma\right)$, $\forall\left(x_{F}, \sigma\right) \in X_{F} \times \Sigma$.

4: Compute automata $G_{N i}, \forall i \in A$, from $G_{N}$ as follows: 4.1: Define function $R_{i}: T R\left(G_{N}\right) \rightarrow \Sigma_{R i}$ as:

$$
R_{i}(x, \sigma)= \begin{cases}\sigma, & \text { if }(x, \sigma) \in \Omega_{i}, \\ \sigma_{R i}, & \text { if }(x, \sigma) \notin \Omega_{i} .\end{cases}
$$

4.2: Construct automata $G_{N i}=\left(X_{N}, \Sigma_{R i}, f_{N i}, x_{0}\right)$, where $\Sigma_{R i}=\left\{R_{i}(x, \sigma):(x, \sigma) \in T R\left(G_{N}\right)\right\}$, and $f_{N i}\left(x, R_{i}(x, \sigma)\right)=f_{N}(x, \sigma), \forall(x, \sigma) \in X_{N} \times \Sigma_{N}$.

5: Compute the verifier automaton $G_{V I}$ from $G_{N i}$ and $G_{F I}$ as: $G_{V I}=A c\left(X_{V I}, \Sigma_{V I}, f_{V I}, x_{0_{V I}}\right)$, where $X_{V I}=X_{N} \times X_{N} \times X_{F}, \Sigma_{V I}=\Sigma_{R 1} \cup \Sigma_{R 2} \cup \Sigma_{F I}$, $x_{0_{V I}}=\left(x_{0}, x_{0},\left(x_{0}, N\right)\right)$, and $f_{V I}: X_{V I} \times \Sigma_{V I} \rightarrow X_{V I}$ is given by Equation (2).

6: Verify the existence of a cyclic path in $G_{V I}, c l=$ $\left(x_{V I_{1}}, \sigma_{1}, x_{V I_{2}}, \sigma_{2}, \ldots, \sigma_{k-1}, x_{V I_{k}}\right)$, with at least one $j \in\{1,2, \ldots, k-1\}$, such that $\sigma_{j} \in \Sigma_{F I}$, and the label of $x_{F}$ in $x_{V I_{j}}=\left(x_{1}, x_{2}, x_{F}\right)$ is equal to $F$. If there exists $c l$ satisfying these conditions, then language $L$ is not codiagnosable w.r.t. $P_{\Omega_{i}}$ and $\Sigma_{f}$. Otherwise, $L$ is codiagnosable w.r.t. $P_{\Omega_{i}}$ and $\Sigma_{f}$.

In Steps 1 and 2 of Algorithm 1, the fault-free automaton $G_{N}$ and the fault automaton $G_{F}$ are computed. In Step 3 , each event $\sigma$ of a transition $\left(x_{F}, \sigma\right)$ of $G_{F}$ is labeled with the indexes of the agents that are able to observe the occurrence of $\sigma$, indicated by function $I\left(x_{F}, \sigma\right)$. In Step 4 , function $R_{i}(x, \sigma)$ renames the unobservable events of $G_{N}$ according to the dynamic observation of the system events of each local agent $i \in A$, making them private events of $G_{N i}$. In Step 5, the E-VERIFIER $G_{V I}$ is computed from $G_{N i}$ and $G_{F I}$, where the transition function $f_{V I}$ is defined in Equation (2). Note that a transition of $G_{V I}$, $\left(\left(x_{1}, x_{2}, x_{F}\right), \sigma^{I\left(x_{F}, \sigma\right)}\right)$, is defined if, and only if, $\sigma^{I\left(x_{F}, \sigma\right)}$ is feasible in state $x_{F}$ of $G_{F I}$, and $\sigma$ is feasible in all states $x_{i}$ of automata $G_{N i}$ indicated by the index function $I\left(x_{F}, \sigma\right)$. A transition $\left(\left(x_{1}, x_{2}, x_{F}\right), \sigma\right)$, where $\sigma$ is a private event of $G_{N i}$, is defined in $G_{V I}$ if it is feasible in $x_{i}$. The 


$$
f_{V I}\left(\left(x_{1}, x_{2}, x_{F}\right), \sigma^{\prime}\right)= \begin{cases}\left(f_{N 1}\left(x_{1}, \sigma\right), f_{N 2}\left(x_{2}, \sigma\right), f_{F I}\left(x_{F}, \sigma^{\prime}\right)\right), & \text { if }\left[\sigma^{\prime}=\sigma^{\{1,2\}}\right] \wedge\left[\sigma \in \Gamma_{G_{N 1}}\left(x_{1}\right)\right] \wedge \\ \left(f_{N 1}\left(x_{1}, \sigma\right), x_{2}, f_{F I}\left(x_{F}, \sigma^{\prime}\right)\right), & \text { if }\left[\sigma^{\prime}=\Gamma_{G^{2}}\left(x_{2}\right)\right], \\ \left(x_{1}, f_{N 2}\left(x_{2}, \sigma\right), f_{F I}\left(x_{F}, \sigma^{\prime}\right)\right), & \text { if }\left[\sigma^{\prime}=\sigma^{\prime 2\}}\right] \wedge\left[\sigma \in \Gamma_{G_{N 1}}\left(x_{1}\right)\right], \\ \left(x_{1}, x_{2}, f_{F I}\left(x_{F}, \sigma^{\prime}\right)\right), & \text { if }\left[\sigma^{\prime}=\sigma^{\emptyset}\right], \\ \left(f_{N 1}\left(x_{1}, \sigma^{\prime}\right), x_{2}, x_{F}\right), & \text { if } \sigma^{\prime} \in \Sigma_{R 1} \backslash \Sigma, \\ \left(x_{1}, f_{N 2}\left(x_{2}, \sigma^{\prime}\right), x_{F}\right), & \text { if } \sigma^{\prime} \in \Sigma_{R 2} \backslash \Sigma, \\ \text { undefined, } & \text { otherwise }\end{cases}
$$

operation presented in Step 5 of Algorithm 1 to compute $G_{V I}$ is called in this paper the indexed synchronous composition. Finally, in Step 6, a cyclic path that violates the codiagnosability of $L$ with dynamic observations is searched. This search can be performed, as in Moreira et al. (2011), by computing the strongly connected components of the E-VERIFIER, whose complexity is linear in its size (Cormen et al., 2000).

Remark 1. Note that in Yin and Lafortune (2015), it is necessary to partition set $A$ of the indexes of the local agents for each possible transition of the T-VERIFIER into four sets, and then, use these sets for defining the feasible transitions. Thus, in the worst-case, it is necessary to partition $A$ into four sets $n \times|X|^{n+1} \times|\Sigma|$ times. As shown in Algorithm 1, the conditions associated with the dynamic observations are added directly to the fault automaton $G_{F I}$ by using the index function $I$, and to the fault-free automata $G_{N i}$ by renaming the unobservable events for each local agent $i \in A$. Thus, this step is carried out with complexity $O(n \times|X| \times|\Sigma|)$, which shows that the E-VERIFIER can be computed more efficiently than the T-VERIFIER when the size of the verifier grows.

It is important to remark that the indexed synchronous composition used for the computation of the E-VERIFIER becomes equal to the standard synchronous composition when dynamic observations are replaced with static observations. In this case, the E-VERIFIER becomes equal to the verifier proposed in Moreira et al. (2011).

The correctness of Algorithm 1 is proved in Theorem 1. In order to do so, it is necessary to introduce first the following definitions. The projection $\theta: \mathcal{L}\left(G_{V I}\right) \rightarrow \Sigma_{F I}^{*}$ is defined recursively as $\theta(\varepsilon)=\varepsilon$, and

$$
\theta(s e)= \begin{cases}\theta(s), & \text { if } e \in \Sigma_{R i} \backslash \Sigma, i \in A \\ \theta(s) e, & \text { otherwise. }\end{cases}
$$

The projection $\theta_{i}: \mathcal{L}\left(G_{V I}\right) \rightarrow \Sigma_{V I}^{*}, i \in A$, is defined recursively as $\theta_{i}(\varepsilon)=\varepsilon$, and, for all $s \in \mathcal{L}\left(G_{V I}\right)$ such that $x_{V I}=f_{V I}\left(x_{0_{V I}}, s\right)=\left(x_{1}, x_{2}, x_{F}\right)$, and $s e \in \mathcal{L}\left(G_{V I}\right)$ with $e \in \Sigma_{V I}$,

$$
\theta_{i}(s e)=\left\{\begin{aligned}
\theta_{i}(s) e, & \text { if }\left[e=\sigma^{I\left(x_{F}, \sigma\right)} \wedge i \in I\left(x_{F}, \sigma\right)\right] \vee \\
& {\left[e \in \Sigma_{R i} \backslash \Sigma\right] } \\
\theta_{i}(s), & \text { otherwise. }
\end{aligned}\right.
$$

The label removing function $R_{l}$ is defined as $R_{l}: \Sigma_{V I} \rightarrow \Sigma$, where $R_{l}(\sigma)=R_{l}\left(\sigma_{R_{i}}\right)=R_{l}\left(\sigma^{I\left(x_{F}, \sigma\right)}\right)=\sigma$. Function $R_{l}$ can be extended to the domain $\Sigma_{V I}^{*}$ as $R_{l}\left(s_{V I} \sigma_{V I}\right)=$ $R_{l}\left(s_{V I}\right) R_{l}\left(\sigma_{V I}\right)$, for all $s_{V I} \in \Sigma_{V I}^{*}$ and $\sigma_{V I} \in \Sigma_{V I}$, and $R_{l}(\varepsilon)=\varepsilon$. Function $R_{l}$ removes all indexes and labels of the events of $\Sigma_{V I}$.

Lemma 1. Let $t \in \mathcal{L}\left(G_{V I}\right)$, then, for all $i \in A$, we have that: $P_{\Omega_{i}}\left[R_{l}(\theta(t))\right]=P_{\Omega_{i}}\left[R_{l}\left(\theta_{i}(t)\right)\right]$.
Proof. The proof will be done by induction in the length of trace $t \in \mathcal{L}\left(G_{V I}\right)$. Without loss of generality we consider that there exist only two local agents, i.e., $A=\{1,2\}$, and the proof will be done with $i=1$. The same procedure can be done for all $i \in A$.

If $\|t\|=0$, i.e., $t=\varepsilon$, then Lemma 1 holds true since $\theta(\varepsilon)=\varepsilon$ and $\theta_{i}(\varepsilon)=\varepsilon$.

Induction hypothesis: Suppose that Lemma 1 is true for $\|t\|=p$, i.e., $P_{\Omega_{1}}\left[R_{l}(\theta(t))\right]=P_{\Omega_{1}}\left[R_{l}\left(\theta_{1}(t)\right)\right]$.

Let te $\in \mathcal{L}\left(G_{V I}\right)$, e $\in \Sigma_{V I}$. Then, $\|t e\|=p+1$, and $P_{\Omega_{1}}\left[R_{l}(\theta(t e))\right]=P_{\Omega_{1}}\left[R_{l}(\theta(t)) R_{l}(\theta(e))\right]$, and $P_{\Omega_{1}}\left[R_{l}\left(\theta_{1}(t e)\right)\right]$ $=P_{\Omega_{1}}\left[R_{l}\left(\theta_{1}(t)\right) R_{l}\left(\theta_{1}(e)\right)\right]$.

There exist six possibilities for event $e$ : $(i) e=\sigma^{\{1,2\}} ;(i i)$ $e=\sigma^{\{1\}} ;($ iii $) e=\sigma^{\{2\}} ;(i v) e=\sigma^{\emptyset} ;(v) e=\sigma_{R 1}$; or $(v i)$ $e=\sigma_{R 2}$.

In cases $(i)$ and $(i i)$, we have that $R_{l}(\theta(e))=\sigma$ and $\left(f_{F}\left(x_{0}, R_{l}(\theta(t))\right), \sigma\right) \in \Omega_{1}$. Then, according to Equation (1), we have that $P_{\Omega_{1}}\left[R_{l}(\theta(t)) R_{l}(\theta(e))\right]=P_{\Omega_{1}}\left[R_{l}(\theta(t))\right] \sigma$. In addition, $R_{l}\left(\theta_{1}(e)\right)=\sigma$ and $\left(f_{N}\left(x_{0}, R_{l}\left(\theta_{1}(t)\right)\right), \sigma\right) \in \Omega_{1}$. Then, we have that $P_{\Omega_{1}}\left[R_{l}\left(\theta_{1}(t)\right) R_{l}\left(\theta_{1}(e)\right)\right]=$ $P_{\Omega_{1}}\left[R_{l}\left(\theta_{1}(t)\right)\right] \sigma$. Thus, by the induction hypothesis, Lemma 1 holds true.

In cases $(i i i)$ and $(i v)$, we have that $R_{l}(\theta(e))=\sigma$ and $\left(f_{F}\left(x_{0}, R_{l}(\theta(t))\right), \sigma\right) \notin \Omega_{1}$. Then, according to Equation (1), we have that $P_{\Omega_{1}}\left[R_{l}(\theta(t)) \sigma\right]=P_{\Omega_{1}}\left[R_{l}(\theta(t))\right]$. In addition, since $R_{l}\left(\theta_{1}(e)\right)=\varepsilon$, then we have that $P_{\Omega_{1}}\left[R_{l}\left(\theta_{1}(t)\right) R_{l}\left(\theta_{1}(e)\right)\right]=P_{\Omega_{1}}\left[R_{l}\left(\theta_{1}(t)\right)\right]$. Thus, by the induction hypothesis, Lemma 1 holds true.

In case $(v)$, we have that $R_{l}(\theta(e))=\varepsilon$. Then, $P_{\Omega_{1}}\left[R_{l}(\theta(t)) R_{l}(\theta(e))\right]=P_{\Omega_{1}}\left[R_{l}(\theta(t))\right]$. In addition, $R_{l}\left(\theta_{1}(e)\right)=\sigma$ but $\left(f_{N}\left(x_{0}, R_{l}\left(\theta_{1}(t)\right)\right), \sigma\right) \notin \Omega_{1}$. Then, $P_{\Omega_{1}}\left[R_{l}\left(\theta_{1}(t)\right) \sigma\right]=P_{\Omega_{1}}\left[R_{l}\left(\theta_{1}(t)\right)\right]$.

In cases $(v i)$, we have that $R_{l}(\theta(e))=R_{l}\left(\theta_{1}(e)\right)=$ $\varepsilon$. Then, $P_{\Omega_{1}}\left[R_{l}(\theta(t)) R_{l}(\theta(e))\right]=P_{\Omega_{1}}\left[R_{l}(\theta(t))\right]$, and $P_{\Omega_{1}}\left[R_{l}\left(\theta_{1}(t)\right) R_{l}\left(\theta_{1}(e)\right)\right]=P_{\Omega_{1}}\left[R_{l}\left(\theta_{1}(t)\right)\right]$. Thus, by the induction hypothesis, Lemma 1 holds true.

Theorem 1. Let $L$ and $L_{N}$ be the languages generated by automata $G$ and $G_{N}$, respectively. Consider the dynamic projections $P_{\Omega_{i}}: L \rightarrow \Sigma_{o_{i}}^{*}, i \in A$, and the set of fault events $\Sigma_{f}=\left\{\sigma_{f}\right\}$. Then, $L$ is not codiagnosable w.r.t. $P_{\Omega_{i}}$ and $\Sigma_{f}$ if, and only if, there exists a cyclic path in $G_{V I}, c l=\left(x_{V I_{1}}, \sigma_{1}, x_{V I_{2}}, \sigma_{2}, \ldots, \sigma_{k-1}, x_{V I_{k}}\right)$, satisfying the following condition:

$\exists j \in\{1,2, \ldots, k-1\}$ such that $\sigma_{j} \in \Sigma_{F I}$, and the label of $x_{F}$ in $x_{V I_{j}}=\left(x_{1}, x_{2}, x_{F}\right)$ is equal to $F$.

Proof. $(\Leftarrow)$ Suppose that there exists a cyclic path $c l$ in $G_{V I}$, satisfying condition (3). Since the label of $x_{F}$ in $x_{V I_{j}}=\left(x_{1}, x_{2}, x_{F}\right)$ is equal to $F$, then, by construction 


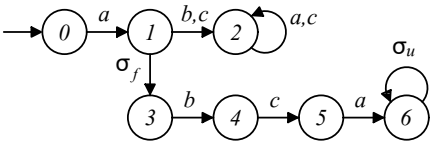

(a)
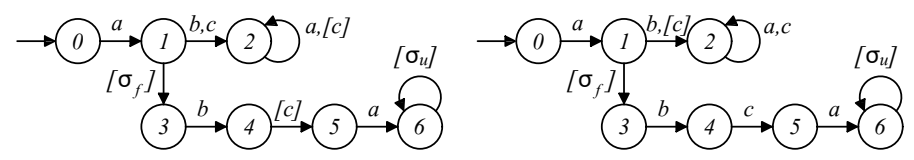

(b)

Figure 1. (a) Automaton $G$ (b) Transition-based observations for Agent 1 and 2, respectively.

of $G_{V I}$, the labels of $x_{F}$ in $x_{V I_{j}}$ will be $F$, for all $j \in\{1, \ldots, k-1\}$. This implies that there exists a trace of events $s_{V I} t_{V I} \in \mathcal{L}\left(G_{V I}\right)$ such that $\sigma_{f} \in s_{V I}$, and $t_{V I}=\left(\sigma_{1}, \ldots, \sigma_{k-1}\right)^{p}, p \in \mathbb{N}$ and $\left\|t_{V I}\right\|>n, \forall n \in \mathbb{N}$.

Define the traces $s_{f}=R_{l}\left(\theta\left(s_{V I} t_{V I}\right)\right) \in \mathcal{L}(G), \omega_{1}=$ $R_{l}\left(\theta_{1}\left(s_{V I} t_{V I}\right)\right) \in \mathcal{L}\left(G_{N}\right)$ and $\omega_{2}=R_{l}\left(\theta_{2}\left(s_{V I} t_{V I}\right)\right) \in$ $\mathcal{L}\left(G_{N}\right)$. According to Lemma 1 , we have that $P_{\Omega_{1}}\left[R_{l}\left(\theta\left(s_{V I} t_{V I}\right)\right)\right]=P_{\Omega_{1}}\left[R_{l}\left(\theta_{1}\left(s_{V I} t_{V I}\right)\right)\right]$, and $P_{\Omega_{2}}\left[R_{l}\left(\theta\left(s_{V I} t_{V I}\right)\right)\right]=P_{\Omega_{2}}\left[R_{l}\left(\theta_{2}\left(s_{V I} t_{V I}\right)\right)\right]$, i.e., $P_{\Omega_{1}}\left(s_{f}\right)=$ $P_{\Omega_{1}}\left(\omega_{1}\right)$ and $P_{\Omega_{2}}\left(s_{f}\right)=P_{\Omega_{2}}\left(\omega_{2}\right)$. Since, according to condition (3), there exists $j \in\{1,2, \ldots, k-1\}$ such that $\sigma_{j} \in \Sigma_{F I}$, then $\left\|\theta\left(t_{V I}\right)\right\|>n^{\prime}, \forall n^{\prime} \in \mathbb{N}$. Thus, trace $s_{f}$ has an arbitrarily long length after the occurrence of $\sigma_{f}$, which leads, according to Definition 2, to the violation of codiagnosability with dynamic observations of $L$.

$(\Rightarrow)$ Suppose that $L$ is not codiagnosable w.r.t. $P_{\Omega_{1}}$, $P_{\Omega_{2}}$ and $\Sigma_{f}$. Then, there exists at least one trace $s_{f}=$ st $\in \mathcal{L}\left(G_{F}\right)$, where $\sigma_{f} \in s$ and $\|t\|>n, \forall n \in \mathbb{N}$, and $\omega_{1}, \omega_{2} \in \mathcal{L}\left(G_{N}\right)$, such that $P_{\Omega_{1}}\left(s_{f}\right)=P_{\Omega_{1}}\left(\omega_{1}\right)$ and $P_{\Omega_{2}}\left(s_{f}\right)=P_{\Omega_{2}}\left(\omega_{2}\right)$. According to the construction of the E-VERIFIER, there exists a sequence $s_{V I} t_{V I} \in$ $\mathcal{L}\left(G_{V I}\right)$ such that $s=R_{l}\left(\theta\left(s_{V I}\right)\right), t=R_{l}\left(\theta\left(t_{V I}\right)\right), \omega_{1}=$ $R_{l}\left(\theta_{1}\left(s_{V I} t_{V I}\right)\right)$, and $\omega_{2}=R_{l}\left(\theta_{2}\left(s_{V I} t_{V I}\right)\right)$. Since $\sigma_{f} \in s$, then all components $x_{F}$ of the states $\left(x_{N 1}, x_{N 2}, x_{F}\right)$ of $G_{V I}$ are labeled with $F$ after the occurrence of trace $s_{V I}$. In addition, since $t=R_{l}\left(\theta\left(t_{V I}\right)\right)$, and $\|t\| \geq n, \forall n \in \mathbb{N}$, then there exists in $G_{V I}$ a cyclic path $c l$ associated with trace $t_{V I}$ where at least one of the events that form $\mathrm{cl}$ belongs to $\Sigma_{F I}$, which concludes the proof.

Example 1. Let $G$ be the automaton model of the system depicted in Fig. 1(a), with the transition-based dynamic observations presented in Fig. 1(b) for Agents 1 and 2, respectively, where the unobservable events are represented in brackets. Thus, we have that $\Omega_{1}=$ $\{(0, a),(1, b),(1, c),(2, a),(3, b),(5, a)\}$ and $\Omega_{2}=\{(0, a)$, $(1, b),(2, a),(2, c),(3, b),(4, c),(5, a)\}$, for Agents 1 and 2, respectively.

According to Steps 1 and 2 of Algorithm 1, and assuming $\sigma_{f}$ as the unique fault event, we obtain automaton $G_{N}$ that models the fault-free behavior of the system, and $G_{F}$ that models the fault behavior of the system, depicted in Figures 2(a) and 2(b), respectively.

In Step 3 automaton $G_{F I}$ is computed by adding the local agents indexes to the events of $G_{F}$ according to the dynamic observations represented in the sets $\Omega_{1}$ and $\Omega_{2}$, as shown in Figure $3(\mathrm{a})$. In Step 4 automata $G_{N 1}$ and $G_{N 2}$ are computed by renaming the events of $G_{N}$ that

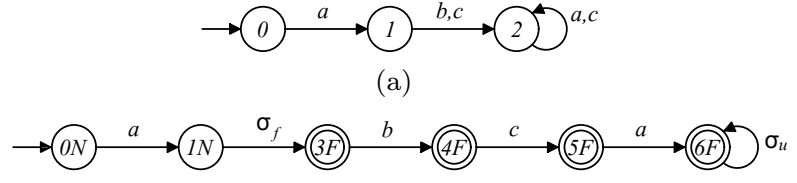

(b)

Figure 2. (a) Automaton $G_{N}$ (b) Automaton $G_{F}$.

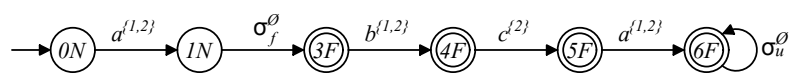

(a) Automaton $G_{F I}$

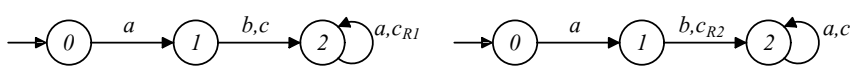

(b) Automaton $G_{N 1}$

(c) Automaton $G_{N 2}$

Figure 3. Automata required to obtain $G_{V I}$.

are unobservable by their respective agents. Automata $G_{N 1}$ and $G_{N 2}$ are depicted in Figures $3(\mathrm{~b})$ and $3(\mathrm{c})$, respectively. The computation of $G_{V I}$ is obtained in Step 5 of Algorithm 1. The initial state of $G_{V I}$ is defined as $(0,0,0 N)$. Then, according to the transition function given by Equation (2), it is necessary to find the events that are feasible in each state $\left(x_{1}, x_{2}, x_{F}\right)$ of $G_{V I}$. If event $\sigma^{\{1,2\}}$ is feasible in state $x_{F}$, and $\sigma$ is feasible in $x_{1}$ and $x_{2}$, then transition $\left(\left(x_{1}, x_{2}, x_{F}\right), \sigma^{\{1,2\}}\right)$ in $G_{V I}$ is defined. If event $\sigma^{\{1\}}$ (resp. $\sigma^{\{2\}}$ ) is feasible in $x_{F}$, it is necessary to check if $\sigma$ is active in $x_{1}$ (resp. $x_{2}$ ). If it is true, then transition $\left(\left(x_{1}, x_{2}, x_{F}\right), \sigma^{\{1\}}\right)$ (resp. $\left.\left(\left(x_{1}, x_{2}, x_{F}\right), \sigma^{\{2\}}\right)\right)$ is defined in $G_{V I}$. If event $\sigma^{\emptyset}$ is feasible in $x_{F}$, then transition $\left(\left(x_{1}, x_{2}, x_{F}\right), \sigma^{\emptyset}\right)$ is defined. Finally, if the private event $\sigma_{R i}$ is feasible in $x_{i}, i \in\{1,2\}$, transition $\left(\left(x_{1}, x_{2}, x_{F}\right), \sigma_{R i}\right)$ is defined in $G_{V I}$. Automaton $G_{V I}$ is depicted in Figure 4.

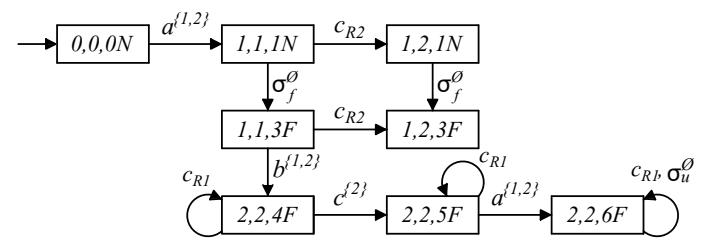

Figure 4. E-VERIFIER $G_{V I}$.

Since $G_{V I}$ has a cyclic path $c l=\left((2,2,6 F), \sigma_{u},(2,2,6 F)\right)$ satisfying the conditions presented in Step 6 of Algorithm 1 , then, according to Theorem 1 , the language generated by $G$ is not codiagnosable w.r.t. $P_{\Omega_{i}}$ and $\Sigma_{f}$. Note that the fault trace $s_{f}=a \sigma_{f} b c a \sigma_{u}^{n}$, for $n \in \mathbb{N}$, has the same dynamic projection than the fault-free trace $s_{N 1}=a b a$, for Agent 1, and the fault-free trace $s_{N 2}=a b c a$, for Agent 2. These traces are associated with trace $t=$ $a^{\{1,2\}} \sigma_{f}^{\emptyset} b^{\{1,2\}} c^{\{2\}} a^{\{1,2\}}\left(\sigma_{u}^{\emptyset}\right)^{n}$ of $G_{V I}$ that ends with the cyclic path $c l$.

\section{COMPLEXITY ANALYSIS OF ALGORITHM}

The computational complexity of Algorithm 1 can be obtained considering that the existence of cyclic paths described in Step 6 is a problem of finding all strongly connected components of $G_{V I}$. Thus, the computational complexity of verifying codiagnosability is linear with respect to the number of states and transitions of $G_{V I}$ (Cormen et al., 2000). 
Table 1 shows the maximum number of states and transitions of all automata that must be computed in order to obtain the E-VERIFIER $G_{V I}$ for $n$ local agents according to Algorithm 1. The computation of the number of states and transitions for automata $G_{N}$ and $G_{F}$ can be seen in Moreira et al. (2011). In the third step of Algorithm 1 , we obtain automaton $G_{F I}$ by adding the indexes of the local agents that observe the transitions of $G_{F}$ to its events. Thus, $G_{F I}$ has the same number of states and transitions than $G_{F}$. In the fourth step automata $G_{N i}$, for $i \in A$, are obtained from $G_{N}$ by renaming the unobservable events according to function $R_{i}$, creating $n$ automata with the same number of states and transitions than $G_{N}$. Finally, in the fifth step, automaton $G_{V I}$ is obtained by making the indexed synchronous composition whose transition function is presented in Equation (2). Since the number of states of $G_{N i}$ and $G_{F I}$ are $|X|$ and $2|X|$, respectively, the number of states of $G_{V I}$ is, in the worst-case, equal to $2|X|^{n+1}$. Furthermore, since for each state of $G_{V I}$ there may exist $|\Sigma|+n\left(|\Sigma|-\left|\Sigma_{f}\right|\right)$ feasible events, the number of transitions of $G_{V I}$ is, in the worstcase, equal to $2|X|^{n+1}\left[|\Sigma|+n\left(|\Sigma|-\left|\Sigma_{f}\right|\right)\right]$. Therefore, the computational complexity of Algorithm 1 is $O\left(n \times|X|^{n+1} \times\right.$ $|\Sigma|)$. Thus, the complexity of computing the E-VERIFIER is the same of the T-VERIFIER presented in Yin and Lafortune (2015), although it may have less states than the T-VERIFIER since it searches for traces of $G_{F}$ and $G_{N}$ with the same dynamic projection, whereas the TVERIFIER searches for traces in $G$ and $G_{N}$ with the same dynamic projection. In addition, the evaluation of which local agents are able to observe dynamically an event is avoided in the E-VERIFIER for each one of its transitions as explained in Remark 1.

Table 1. Complexity Analysis of Algorithm 1

\begin{tabular}{c|c|c}
\hline & $N^{o}$ of States & $N^{o}$ of Transitions \\
\hline$G$ & $|X|$ & $|X| \times|\Sigma|$ \\
\hline$G_{N}$ & $|X|$ & $|X|\left(|\Sigma|-\left|\Sigma_{f}\right|\right)$ \\
\hline$G_{F}$ & $2|X|$ & $2|X| \times|\Sigma|$ \\
\hline$G_{F I}$ & $2|X|$ & $|X|\left(|\Sigma|-\left|\Sigma_{f}\right|\right)$ \\
\hline$G_{N i}$ & $|X|$ & $2|X|^{n+1}\left[|\Sigma|+n\left(|\Sigma|-\left|\Sigma_{f}\right|\right)\right]$ \\
\hline$G_{V I}$ & $2|X|^{n+1}$ & $O\left(n \times|X|^{n+1} \times|\Sigma|\right)$ \\
\hline \multicolumn{2}{c}{ Complexity }
\end{tabular}

\section{CONCLUSIONS}

In this paper, we propose an efficient algorithm for the verification of codiagnosability of the language of a DES with dynamic observations. The proposed verifier, called E-VERIFIER, extends to the case of dynamic observations another verifier presented for the case of static observations. In order to obtain the E-VERIFIER, a new synchronous composition is defined, called indexed synchronous composition, that becomes equal to the standard synchronous composition when dynamic projections are replaced with static projections. We show that the EVERIFIER can be computed more efficiently than another verifier proposed in the literature when the size of the verifier grows.

\section{REFERENCES}

Cabral, F.G., Moreira, M.V., Diene, O., and Basilio, J.C. (2015). A Petri net diagnoser for discrete event systems modeled by finite state automata. IEEE Trans. on Automatic Control, 60(1), 59-71.

Cassandras, C.G. and Lafortune, S. (2008). Introduction to Discrete Event Systems. Springer, New York, 2 edition. Cassez, F. and Tripakis, S. (2008). Fault diagnosis with static and dynamic observers. Fundamenta Informaticae, 88(4), 497-540.

Contant, O., Lafortune, S., and Teneketzis, D. (2006). Diagnosability of discrete event systems with modular structure. Discrete Event Dynamic Systems, 16(1), 937.

Cormen, T.H., Leiserson, C.E., and Rivest, R.L. (2000). Introduction to Algorithms. MA: MIT Press, Cambridge, 25 edition.

Debouk, R., Lafortune, S., and Teneketzis, D. (2000). Coordinated decentralized protocols for failure diagnosis of discrete event systems. Discrete Event Dynamic Systems: Theory and Applications, 10(1), 33-86.

Jiang, S., Huang, Z., Chandra, V., and Kumar, R. (2001). A polynomial algorithm for testing diagnosability of discrete-event systems. IEEE Transactions on Automatic Control, 46(8), 1318-1321.

Kilic, E. (2008). Diagnosability of fuzzy discrete event systems. Information Sciences, 178(3), 858-870.

Moreira, M.V., Jesus, T.C., and Basilio, J.C. (2011). Polynomial time verification of decentralized diagnosability of discrete event systems. IEEE Transactions on Automatic Control, 56(7), 1679-1684.

Qiu, W. and Kumar, R. (2006). Decentralized failure diagnosis of discrete event systems. IEEE Transactions on Systems, Man, and Cybernetics Part A:Systems and Humans, 36(2), 384-395.

Sampath, M., Sengupta, R., Lafortune, S., Sinnamohideen, K., and Teneketzis, D. (1995). Diagnosability of discreteevent systems. IEEE Transactions on Automatic Control, 40(9), 1555-1575.

Thorsley, D. and Teneketzis, D. (2007). Active acquisition of information for diagnosis and supervisory control of discrete event systems. Discrete Event Dynamic Systems: Theory and Applications, 17(4), 531-583.

Wang, W., Girard, A.R., Lafortune, S., and Lin, F. (2011). On codiagnosability and coobservability with dynamic observations. IEEE Transactions on Automatic Control, $56(7), 1551-1566$.

Wang, W., Lafortune, S., Girard, A.R., and Lin, F. (2010a). Optimal sensor activation for diagnosing discrete event systems. Automatica, 46(7), 1165-1175.

Wang, W., Lafortune, S., Lin, F., and Girard, A.R. (2010b). Minimization of dynamic sensor activation in discrete event systems for the purpose of control. IEEE Transactions on Automatic Control, 55(11), 2447-2461. Yin, X. and Lafortune, S. (2015). Codiagnosability and coobservability under dynamic observations: Transformation and verification. Automatica, 61, 241-252.

Yoo, T.S. and Lafortune, S. (2002). Polynomial-time verification of diagnosability of partially observed discreteevent systems. IEEE Transactions on Automatic Control, 47(9), 1491-1495.

Zad, S.H., Kwong, R., and Wonham, W. (2005). Fault diagnosis in discrete-event systems: Incorporating timing information. IEEE Transactions on Automatic Control, 50(7), 1010-1015. 